\title{
Status of mirror segment production for the Giant Magellan Telescope
}

\author{
H. M. Martin ${ }^{\mathrm{a}}$, J. H. Burge, ${ }^{\mathrm{a}, \mathrm{b}}$, J. M. Davis ${ }^{\mathrm{a}}$, D. W. Kim ${ }^{\mathrm{b}}$, J. S. Kingsley ${ }^{\mathrm{a}}$, K. Law ${ }^{\mathrm{a}}$, A. Loeff ${ }^{\mathrm{a}}$, R. D. \\ Lutz $^{\mathrm{a}}$, C. Merrill ${ }^{\mathrm{a}}$, P. A. Strittmatter ${ }^{\mathrm{a}}$, M. T. Tuell ${ }^{\mathrm{a}}$, S. N. Weinberger ${ }^{\mathrm{a}}$ and S. C. West ${ }^{\mathrm{a}}$ \\ ${ }^{a}$ Steward Observatory, University of Arizona, Tucson, AZ 85721, USA \\ ${ }^{\mathrm{b}}$ College of Optical Sciences, University of Arizona, Tucson, AZ 85721, USA
}

\begin{abstract}
The Richard F. Caris Mirror Lab at the University of Arizona is responsible for production of the eight $8.4 \mathrm{~m}$ segments for the primary mirror of the Giant Magellan Telescope, including one spare off-axis segment. We report on the successful casting of Segment 4, the center segment. Prior to generating the optical surface of Segment 2, we carried out a major upgrade of our $8.4 \mathrm{~m}$ Large Optical Generator. The upgrade includes new hardware and software to improve accuracy, safety, reliability and ease of use. We are currently carrying out an upgrade of our $8.4 \mathrm{~m}$ polishing machine that includes improved orbital polishing capabilities. We added and modified several components of the optical tests during the manufacture of Segment 1, and we have continued to improve the systems in preparation for Segments 2-8. We completed two projects that were prior commitments before GMT Segment 2: casting and polishing the combined primary and tertiary mirrors for the LSST, and casting and generating a $6.5 \mathrm{~m}$ mirror for the Tokyo Atacama Observatory.
\end{abstract}

Keywords: Giant Magellan Telescope, telescopes, optical fabrication, optical testing, off-axis, aspheres

\section{INTRODUCTION}

The $25 \mathrm{~m}$ GMT primary mirror consists of seven $8.4 \mathrm{~m}$ lightweight honeycomb mirrors ${ }^{[1],[2]}$ This minimally segmented primary mirror, together with the segmented adaptive secondary mirror, guarantees smooth wavefronts over large fractions of the aperture and simplifies alignment and phasing of the telescope. The $8.4 \mathrm{~m}$ segments are being made at the Richard F. Caris Mirror Lab at the University of Arizona. The first segment, one of the seven (including one spare) identical off-axis segments, is finished, and Segments 2-5 are in different stages of manufacture. The off-axis segments present significant challenges in fabrication and optical testing. The interferometric test has a 3-element off-axis null corrector that includes a $3.75 \mathrm{~m}$ curved mirror and has overall dimensions exceeding $8 \mathrm{~m} \cdot{ }^{[3],[4]}$ To mitigate the risk of a mistake in the interferometric test, we have independent measurements of critical parameters including radius of curvature, off-axis geometry and low-order aberrations (astigmatism, coma, trefoil and spherical aberration). These tests are housed in a $28 \mathrm{~m}$ test tower that was built for the GMT segments. Manufacture of Segment 1 demonstrated the essential technology for all the segments. We improved the methods and equipment for fabrication and testing during the manufacture of Segment 1, and we continue to refine them as we start work on the optical surface of Segment 2.

\section{STATUS OF SEGMENT PRODUCTION}

GMT Segment 1, the first off-axis segment, was completed in August 2012. ${ }^{[5],[6]}$ The surface is accurate to $18 \mathrm{~nm}$ rms after a simulated active-optics correction using 27 bending modes with $21 \mathrm{~N} \mathrm{rms}$ correction force. The off-axis geometry meets its tolerances with the error in off-axis distance $=+0.2 \pm 1.2 \mathrm{~mm}$ and the clocking error $=0 \pm 13$ arcseconds. The clocking reference is defined by the loadspreaders bonded to the back of the mirror.

Segments 2 and 3 are also off-axis segments. Segment 2 was cast in January 2012. We have completed work on the rear surface, including bonding the 165 loadspreaders that form the interface between the mirror and its support system. The next step is generating the optical surface, which awaited completion of an upgrade to the generator (Section 4) and generating of the $6.5 \mathrm{~m}$ mirror for the Tokyo Atacama Observatory (TAO). Segment 3 was cast in August 2013. We recently completed grinding and polishing the rear surface in preparation for bonding the loadspreaders.

Segment 4, the center segment, was cast in September 2015. Figure 1 shows the mirror, still on the furnace hearth, after the casting. Section 3 describes the casting and manufacturing plan, including issues unique to the center segment. We are preparing to cast Segment 5 with a tentative date of January 2017. We have purchased the 20 tons of Ohara

Advances in Optical and Mechanical Technologies for Telescopes and Instrumentation II, edited by Ramón Navarro, James H. Burge, Proc. of SPIE Vol. 9912, 99120V

(C) 2016 SPIE · CCC code: 0277-786X/16/\$18 · doi: 10.1117/12.2234491 
Corporation's E6 borosilicate glass for Segment 6. In the near future we will begin the procurement process to obtain the ceramic fiber mold parts for Segment 6 from Rex Materials and the E6 glass for Segment 7.

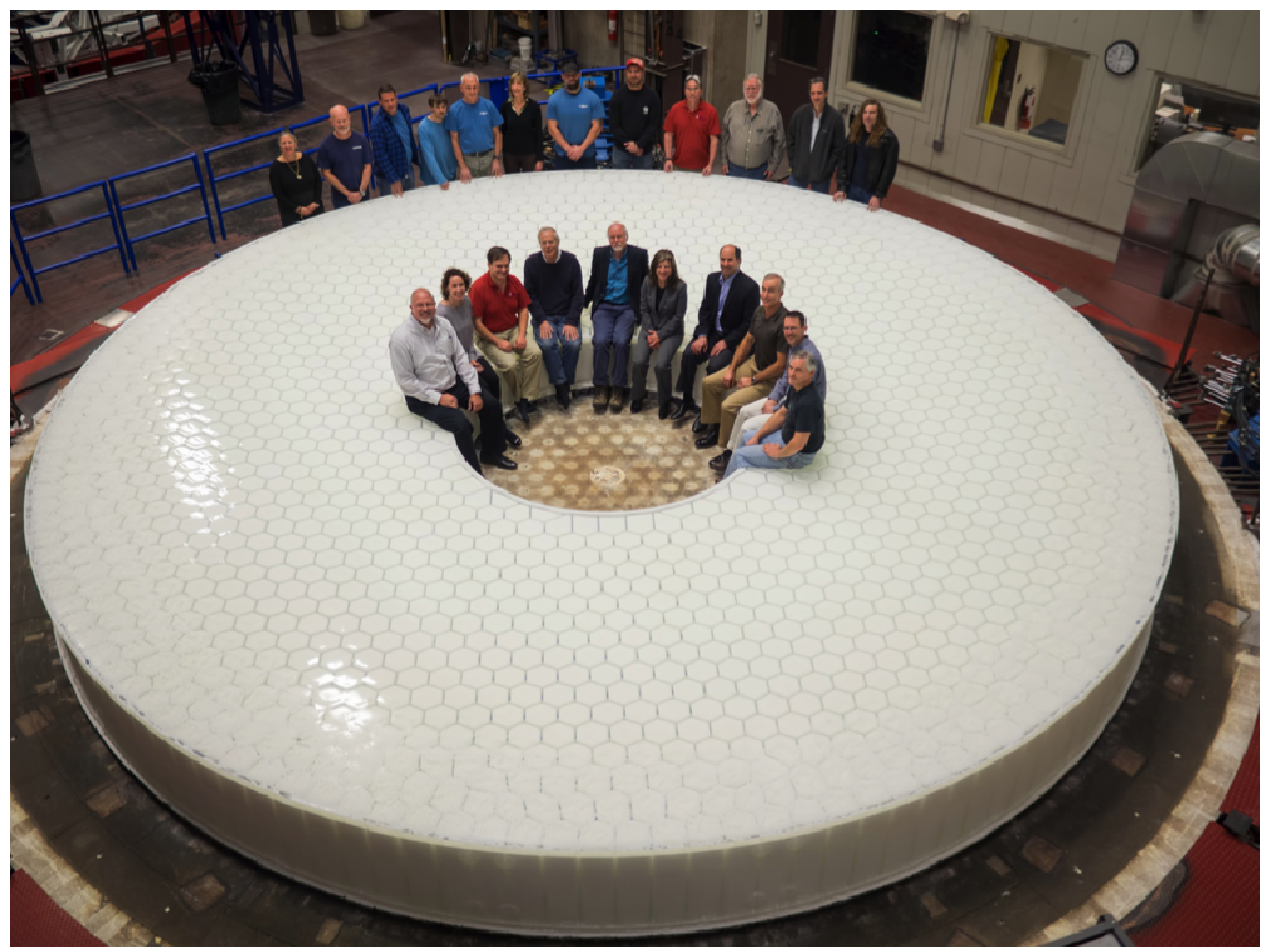

Figure 1. GMT center segment resting on the furnace hearth after the casting. The $2.4 \mathrm{~m}$ Cassegrain hole comfortably seats ten. At the time of the photo the mirror still contained the 1530 ceramic fiber boxes that create the honeycomb structure.

They have since been removed.

Between GMT Segments 1 and 2 we cast the $8.4 \mathrm{~m}$ combined primary and tertiary mirror for the Large Synoptic Survey Telescope (LSST) and the $6.5 \mathrm{~m}$ primary mirror for the TAO. Work on these mirrors accounts for much of the interval between Segments 1 and 2. We finished polishing the LSST mirrors in October 2014. ${ }^{[7]}$ We generated the TAO mirror, which will be polished with a new $6.5 \mathrm{~m}$ machine at the University of Arizona College of Optical Sciences.

\section{MANUFACTURE OF THE CENTER SEGMENT}

The center segment is the fourth segment in the manufacturing sequence. Manufacturing the segments in this order supports the option of an early commissioning of GMT with a close-packed array of four segments. Alignment and phasing are expected to be easier with maximum connections among neighboring segments.

\subsection{Casting the center segment}

The center segment has an inner diameter of $2.4 \mathrm{~m}$, which accommodates the 20 arcminute field and other constraints of the telescope design and is compatible with the layout of the honeycomb mold. The circular boundary breaks the repeating pattern of hexagonal cores; its precise diameter was chosen to optimize the sizes and shapes of partial cores at the boundary.

This is the largest center hole we have cast and it presented challenges to the mold design. The strength of the mold comes from cast silicon carbide cement in the form of an outer cylindrical wall, hexagonal floor tiles, and (for the center segment) an inner cylinder. During the casting, the inner wall of the mold is in compression from the hydrostatic force of liquid glass and is vulnerable to shifting of the sections of the wall. The outer wall, which is being pushed out by the glass, is well constrained from the outside by steel bands in tension, and from the inside by the floor of the mold. It is more difficult to provide a secure constraint for the inner wall. To increase the resistance to shifting of the wall sections, 
we placed a ring of silicon carbide inside the wall about $30 \mathrm{~cm}$ above the floor of the mold, shown in Figure 2. This system was successful at avoiding leaks and keeping the inner edge of the mirror round.

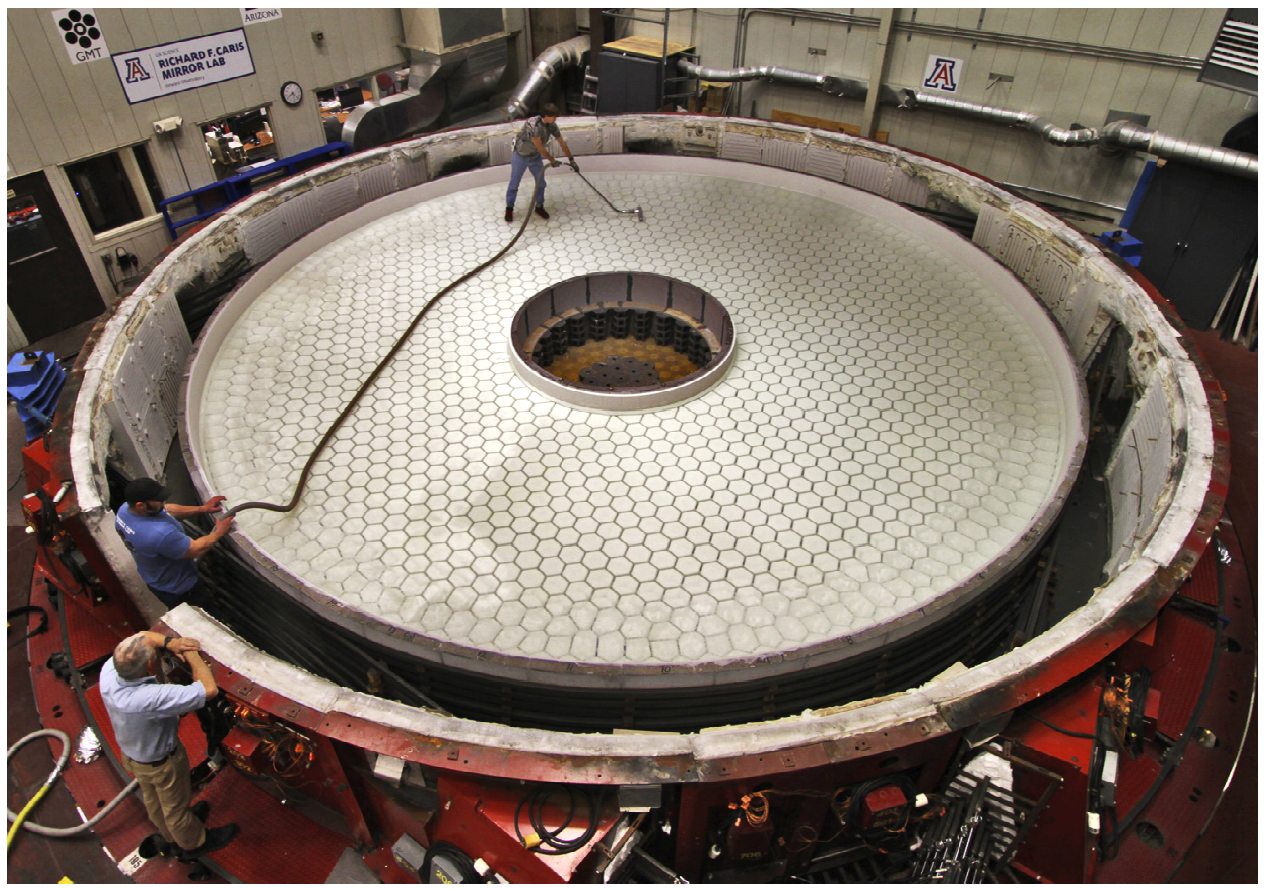

Figure 2. This image of GMT Segment 4 soon after the furnace was opened shows the custom inner tub wall. The silicon carbide cylinder is braced by a ring of silicon carbide.

For every casting, a special support system comes into play as the mirror solidifies and cools. The floor of the furnace bows by $6 \mathrm{~mm}$ due to the vertical temperature gradient while the glass melts, then flattens as the whole structure cools. Once the glass is solid, the flattening tends to shift the mirror support from a uniform distribution to a concentration at the outer edge. We maintain a relatively uniform support by applying forces of about $3000 \mathrm{~N}$ with counterweights at about 70 locations over the interior of the mirror. These pushers support about $2 / 3$ of the weight, leaving $1 / 3$ supported by the furnace floor at the outer edge of the mirror. This "hot float" system was analyzed and redesigned for the very different mass distribution of the center segment. The analysis showed that the maximum mechanical stress in the mirror would be well under the conservative limit of $690 \mathrm{kPa}(100 \mathrm{psi})$.

Other changes required for the center segment include the lifting fixture and the mirror support cell for polishing and testing. The lifting fixture is a steel frame with 36 steel disks that are bonded to the front surface of the mirror with compliant adhesive. (After the mirror is polished, the same fixture is used with vacuum pads instead of adhesive.) The center segment required a new lifting fixture, shown in Figure 3, because of the large center hole and different mass distribution. The mirror support cell will be modified before the center segment is installed. A cover plate will be installed over the unused part of the cell, as well as a large funnel to drain coolant, ground glass, and abrasive slurries during the grinding and polishing operations. 


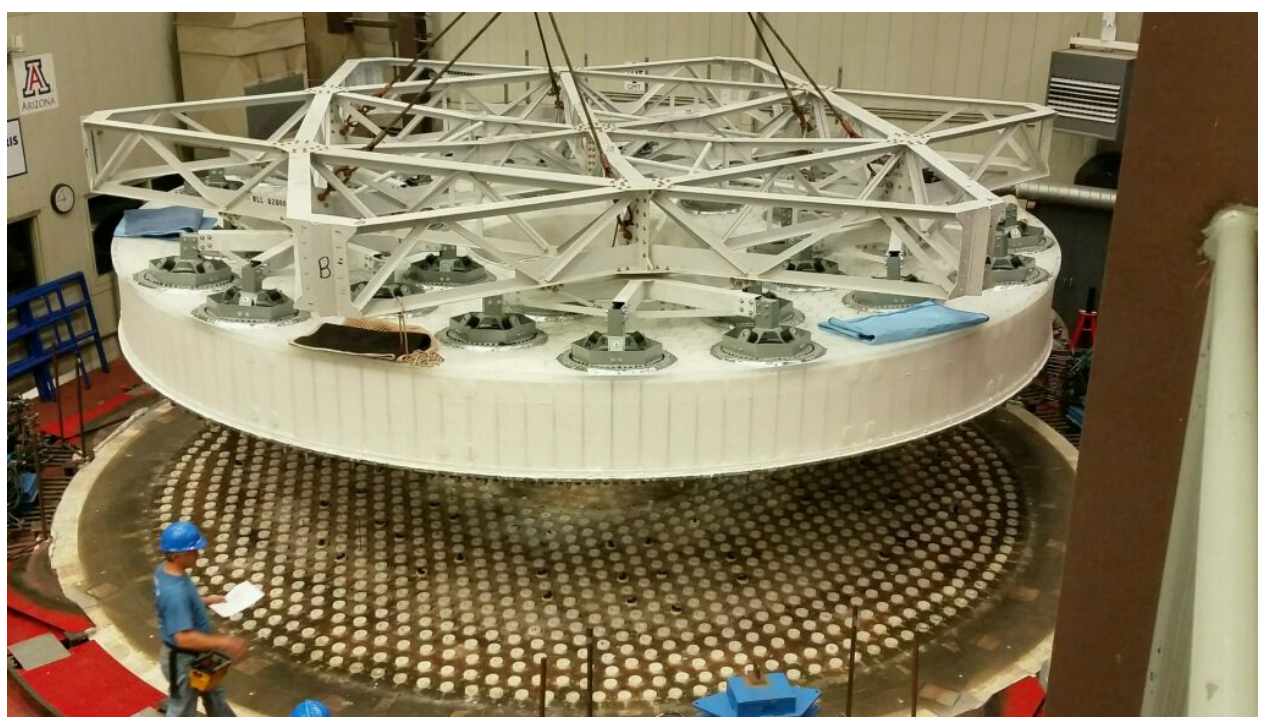

Figure 3. GMT center segment being lifted from the hearth. The steel lifting fixture contains 36 disks that are bonded to the front surface of the mirror with compliant adhesive.

\subsection{Measuring the center segment}

The most significant modifications for the center segment are the optical tests, which are currently configured for the offaxis segments. There are four major tests, which together guide the figuring through all stages of fabrication, demonstrate compliance with all specifications, and provide independent measurements of all critical parameters.

- The interferometric test measures the full aperture as well as radius of curvature to high accuracy. ${ }^{[3]}$ It measures all spatial scales to accuracies that meet the segment specifications, as long as the slope errors are within the capture range of the interferometer. Large-scale accuracy of the test is very sensitive to alignment of the segment and the elements of the null corrector. ${ }^{[4]}$

- The Software Configurable Optical Test System (SCOTS) measures slopes over the full aperture, with high spatial resolution and a very high dynamic range for slope errors. ${ }^{[8],[9]}$ The slopes are integrated to give a full surface measurement with high accuracy on scales up to about $2 \mathrm{~m}$.

- The scanning pentaprism test is the main independent verification of off-axis geometry and large-scale figure. ${ }^{[10],[11],[12]}$ It uses the natural telescope geometry, illuminating the segment with a narrow collimated beam parallel to the telescope's optical axis, which the segment focuses on a detector at the telescope's prime focus. The collimated beam is scanned across the segment by moving a pentaprism along a rail perpendicular to the optical axis. Except for a small correction for the fact that the primary mirror is not parabolic $(k=-0.9983)$ the focused spot should not move as the beam is scanned, and the measured spot motion is proportional to slope errors on the segment. Model fitting gives the radius of curvature, astigmatism, coma, trefoil and spherical aberration.

- The Laser Tracker Plus (LT+) system is a scan of the full surface with a laser tracker, plus stability references to eliminate the effects of rigid-body motion. ${ }^{[13],[14]} \mathrm{LT}+$ works with a ground or polished surface. With a custom calibration of the tracker's angle measurements, it is accurate to $1 \mu \mathrm{m}$ rms surface and $0.4 \mathrm{~mm}$ in radius of curvature.

The interferometric test and SCOTS use a $3.75 \mathrm{~m}$ curved mirror, mounted at the top of the test tower, to fold the beam and provide part of the correction for the aspheric wavefront. The null corrector for the off-axis segments consists of this mirror, a smaller curved mirror, and a computer-generated hologram (CGH). For the off-axis segments, the large mirror is tilted (so it generates astigmatism in the wavefront) and the interferometer and the rest of the null corrector are positioned off-axis as shown in Figure 4. 


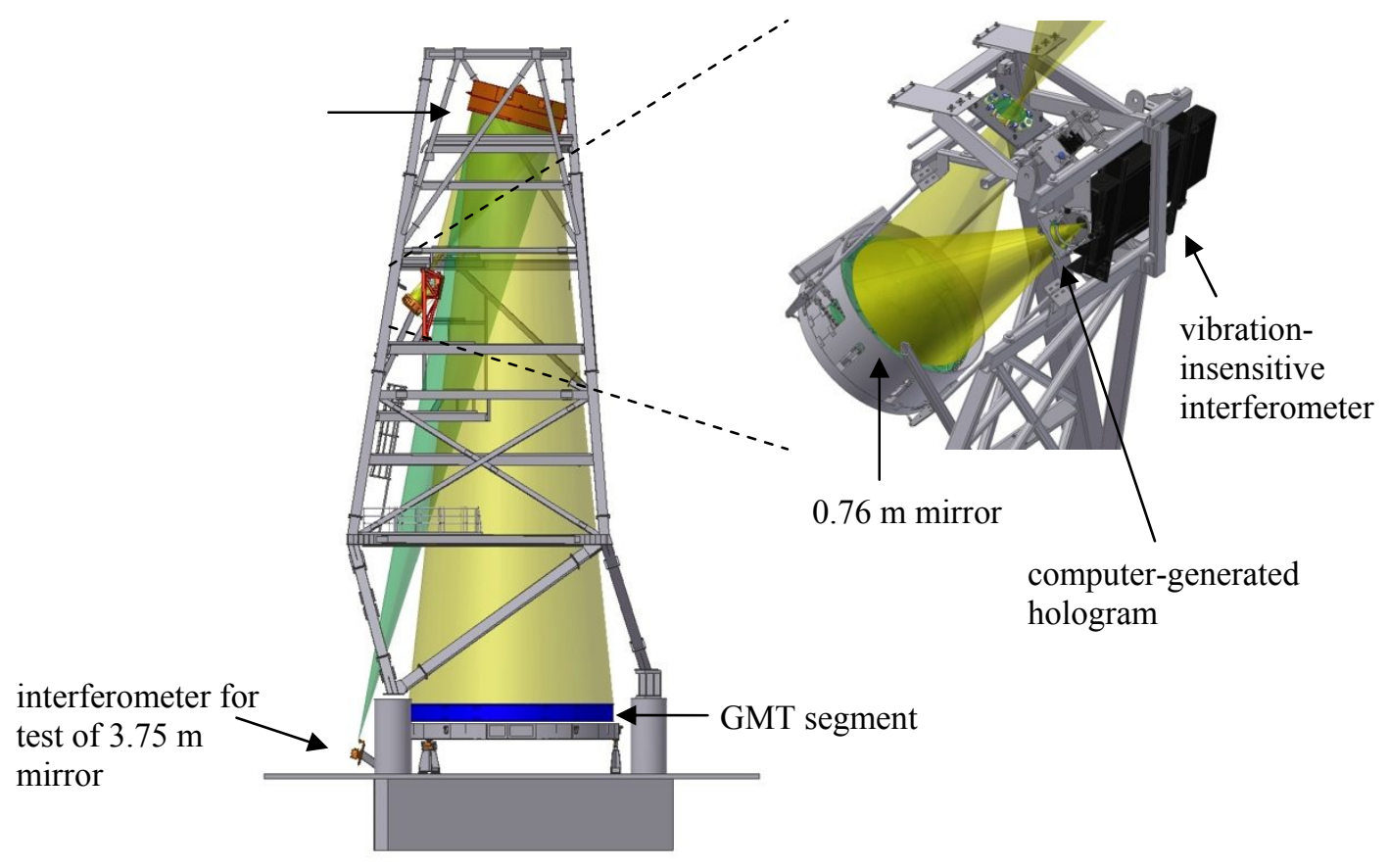

Figure 4. Model of the interferometric test for the GMT off-axis segments, in the test tower. At right is a blow-up of the interferometer and first two elements of the null corrector. Gold light cones represent the measurement of the GMT segment, while the aqua cone in the full model at left represents a simultaneous measurement of the $3.75 \mathrm{~m}$ fold sphere.

We are considering two options for the interferometric test of the axisymmetric center segment. The most natural test system would be centered above the segment. It must use the fold mirror because the test tower does not reach the segment's center of curvature with $R=36 \mathrm{~m}$. The interferometer and additional null optics (at least a CGH) are therefore located between the segment and the fold sphere, and their supporting structure will create obscuration in the test. Furthermore we require a near-simultaneous interferometric test of the $3.75 \mathrm{~m}$ fold mirror because its shape is not perfectly stable; we subtract the fold mirror's shape error from the measured shape of the center segment. The same obscuration affects this measurement of the fold mirror and the subtraction. We can mitigate these effects by rotating the center segment around its axis and making multiple measurements, and by mounting the interferometer and optics in a deployable structure and removing it for the measurement of the fold mirror.

We are also exploring the possibility of eliminating the obscuration by keeping the test in the off-axis position and compensating for the unwanted asymmetric aberrations introduced by the tilted fold mirror. The off-axis test has a second, smaller, curved mirror that can be tilted in a different direction to cancel much of the aberration. In any design, there will be a CGH in the null corrector to create the desired wavefront to roughly $10 \mathrm{~nm}$ accuracy, and it can be designed to correct any wavefront error up to a limiting magnitude of slope error.

The SCOTS test will use the same geometry as the interferometric test, whether it is symmetric or off-axis. The source and detector for SCOTS are located near the approximate focus of the center segment that is created by the fold mirror. SCOTS does not require any particular geometry, but does require accurate knowledge of the geometry.

The equipment for the pentaprism test includes the rail on which the pentaprism is scanned across the diameter, and the detector at the position of the telescope's prime focus. In the lab test of an off-axis segment that faces up, the rail is tilted in a plane that is $13.5^{\circ}$ from horizontal, and the detector is located in the test tower $18 \mathrm{~m}$ above the segment and $4 \mathrm{~m}$ offcenter. For the center segment (also face-up), the rail is horizontal and the detector is centered over the segment. The pentaprism rail is already on a deployable structure, which will be modified to make the rail horizontal, and the detector will require another deployable structure to place it at the telescope focus and remove it to avoid obscuration of the other tests. Accurate alignment, and especially knowledge of the alignment, are critical for all tests. We measure the alignment of all components with laser trackers at the time of the wavefront measurements, so deployable component mounts will not increase measurement errors. 
Physically, the LT + measurement is unchanged for the center segment. Only the data reduction changes with a different ideal surface.

\section{UPGRADE OF LARGE OPTICAL GENERATOR}

We use the Large Optical Generator (LOG) to machine, or generate, each segment to its final dimensions and a surface accuracy of some tens of microns rms. We machine the front and rear surfaces, their edges and bevels, and 48 circular bosses on the outer edge that are potential mounting surfaces for alignment aids. The LOG was first installed at the university's Optical Sciences Center in 1984 and moved to the Mirror Lab in 1990. ${ }^{[15]}$ Some mechanical components were replaced in upgrades over the 30 year life of the machine, but by 2014 a number of subsystems were no longer performing reliably and a more thorough upgrade was needed. We also decided to replace the computer and software architecture, most of which was the original system with C code running under VxWorks on VME computers, although the software had evolved continually over time. The main elements of the LOG upgrade are described below. ${ }^{[16]}$

Our goal for surface accuracy of the remaining GMT segments, after generating, is $10 \mu \mathrm{m}$ rms. This would be a substantial improvement over the roughly $20 \mu \mathrm{m} \mathrm{rms} \mathrm{accuracy}$ we have achieved with axisymmetric mirrors and the 28 $\mu \mathrm{m}$ rms accuracy we achieved with Segment 1, the first non-axisymmetric mirror we generated. (This excludes a larger sag error in Segment 1 due to a measurement issue.) If we can achieve $10 \mu \mathrm{m} \mathrm{rms} \mathrm{accuracy}$ for the generated surface it will reduce the time spent in fine grinding, which is valuable because the fine grinding is done using the polishing machine that defines the critical path for segment production. To generate to $10 \mu \mathrm{m}$ rms for the off-axis segments requires a combination of tool position accuracy, turntable position accuracy and bearing stability, system stiffness, surface measurement accuracy, and feedback of surface measurements into the tool position control. We developed all of these aspects of the LOG system as part of the upgrade. Figure 5 shows the upgraded LOG in the process of generating the rear surface of GMT Segment 3.

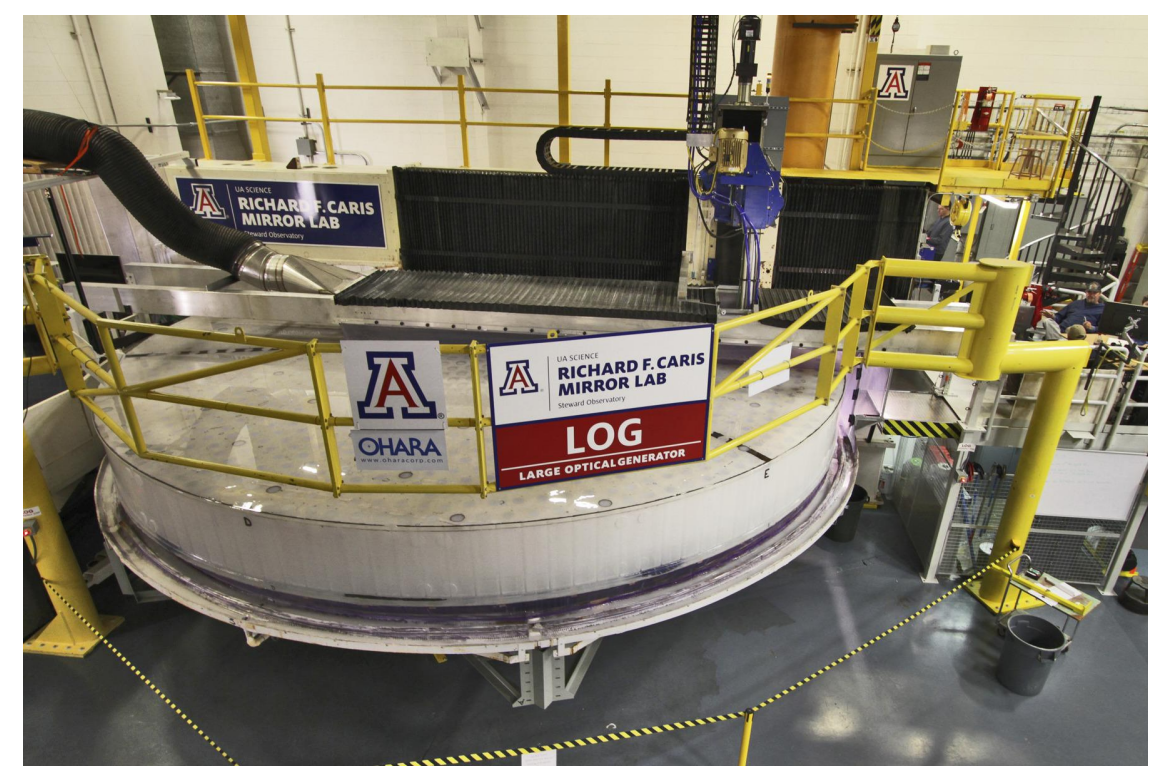

Figure 5. The upgraded LOG generating the flat rear surface of GMT Segment 3. The yellow railing provides fall protection for staff who occasionally work on the mirror.

\subsection{Phased upgrade}

We planned to start the LOG upgrade in 2014 after generating the $6.5 \mathrm{~m}$ TAO mirror. When the horizontal and vertical motor drives started showing signs of failure, we suspended the generating and immediately started the upgrade. We carried it out in three phases, interrupted for partial generating of two mirrors in order to minimize the impact on production. The first and largest phase included most of the hardware upgrades and the basic machine control software, including safety systems. The second phase included enhancements to support generating flat surfaces and culminated with generating a $1.2 \mathrm{~m}$ flat mirror as a test. At its completion in late 2015 , we generated the flat backplate of GMT 
Segment 3. We could then proceed with fine grinding and polishing that surface on the polishing machine while we completed the LOG upgrade. The third phase included software to define tool paths for curved surfaces, both axisymmetric and off-axis surfaces. It also included new turntable encoders, non-contact measurements of surface shape, and a second operator platform. We tested the system by machining a $2 \mathrm{~m}$ granite disk with an asymmetric curved surface requiring about the same range of motion as a GMT off-axis segment. After the third phase we resumed the suspended generating of the TAO mirror. When it is completed in mid-2016 we will generate the front surface of GMT Segment 2.

\subsection{Computers and software}

We replaced the VME real-time control computers and the Sun user interface computer with PCs running new C code in Linux. The new software includes many new features, some of them described in the following sections, and involves a fresh, comprehensive approach to safety and ease of use. The new software automates calibration of the motions and setting the tool position relative to the mirror when a new tool is installed. It also automates safety features such as collision avoidance. It reduces user interaction by automating a sequence of cuts as the tool is stepped from initial cuts on the rough cast surface toward the finished dimension.

\subsection{Turntable gearbox and encoders}

We replaced the gearbox for the turntable that rotates the mirror under the generating tool. To achieve the $10 \mu \mathrm{m}$ rms goal we need accurate and especially repeatable axial runout of the turntable, and accurate control of angular position. The off-axis segments have a maximum tangential slope of $3.3 \mathrm{mrad}$, or $0.25 \mathrm{~mm}$ per degree of turntable motion, at the edge of the mirror. An angular error of $0.04^{\circ}$ causes a $10 \mu \mathrm{m}$ surface error at the edge of the mirror. The original angle encoders were limited by mechanical hysteresis and had errors up to $0.25^{\circ}$. We installed a new pair of encoders directly coupled to the turntable shaft, on opposite sides to reduce sensitivity to shaft displacement. The average of the two signals is accurate to $0.014^{\circ}$ peak-to-valley, as confirmed by laser tracker measurements of a rotating mirror.

\subsection{Motors and ball screws}

We replaced the motors for horizontal and vertical motion, the turntable and the generating spindle. The old horizontal and vertical motors were selected for generating with low speed and high accuracy. We also use the LOG for fine grinding and polishing the flat rear surface of most mirrors, requiring high speed and torque but not high accuracy. In the past we had to change motors between the two operations. The new motors handle the full range of speeds, torques and accuracy required to support generating and polishing. We also replaced the horizontal screw and refurbished the vertical screw because of wear.

\subsection{Surface measurements}

We installed a new non-contact Keyence probe for using the LOG as a profilometer. It attaches to the spindle and uses reflected light to measure the distance to the surface, as the probe is moved along a path that follows the ideal surface. This measurement is safe, convenient, and quick because it is done with the mirror on the LOG. The measurement accuracy is better than the accuracy of the generated surface, because the measurement is not affected by deflections due to cutting force or variable cutting efficiency, but it is subject to errors in motion of the spindle.

To further improve the accuracy, we will make occasional measurements with LT + . The measurement accuracy will be several microns rms, limited by the cusps left on the surface by the generating tool. It requires moving the mirror from the LOG to the test tower then realigning it on the LOG, a time-consuming process. We will use the LT + data to calibrate the LOG profilometer measurements. After calibration, the LOG measurements will be limited by nonrepeatable errors, which we expect to be on the order of $5 \mu \mathrm{m} \mathrm{rms}$. We can maintain this accuracy with very few interruptions for $\mathrm{LT}+$ measurements.

\subsection{Tool path calculations}

We use the multi-purpose SAGUARO software ${ }^{[17]}$ to generate tool paths for the generating runs. Surface cuts are spiral paths with the vertical motion varying to follow the surface prescription. One tool path may be used many times with the vertical position stepping down with successive cuts. Edges and bevels can be generated with small discrete horizontal steps. For cylindrical surfaces (edges of front and rear facesheets, and bosses) we can also use a helical tool path. Tool paths, including speeds, are saved as text files that are read by the LOG control program. 
The surface cuts are made with a cup wheel, a ring of diamond pellets with $r=16 \mathrm{~cm}$ shown in Figure 6 . The spindle is tilted by $\theta=10^{\circ}$ out of the vertical plane, so the lower part of the ring makes contact with the mirror surface. As the tool moves across a curved mirror surface from edge to center, the contact point moves on the tool as it would for a spherical tool with radius $r^{\prime}=r / \sin (\theta)$. To take account of this motion of the contact point, the tool path that the LOG must follow is displaced from the mirror surface by $r^{\prime}$ in the normal direction. For an off-axis GMT segment with $14 \mathrm{~mm}$ peak-to-valley astigmatism, the tool path oscillates vertically with each rotation of the mirror due to the varying surface height, and also oscillates horizontally due to the varying slope (direction of the surface normal).

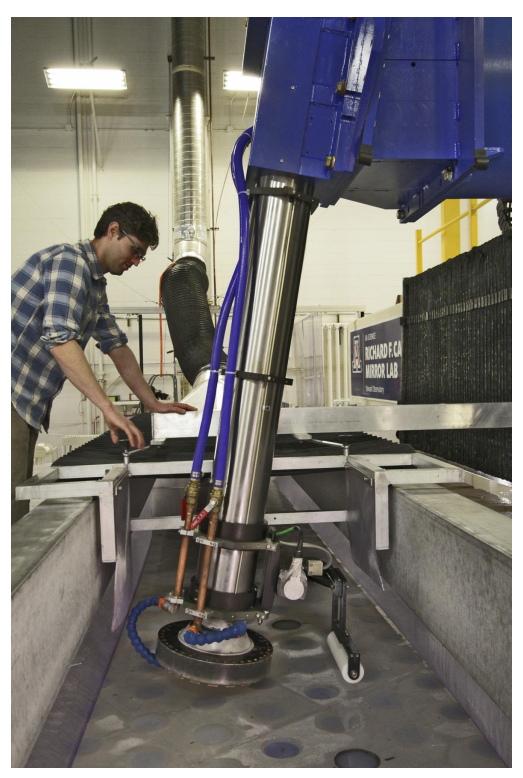

Figure 6. LOG spindle in position to generate the rear surface of Segment 3.

The generated surface never exactly matches the desired surface. Errors in the tool motion, deflection of the mirror under the force of the tool, thermal expansion of the spindle, and variable cutting efficiency all contribute to surface errors that can be significant compared with the $10 \mu \mathrm{m}$ rms accuracy goal. We can use the calibrated surface measurements to make an empirical adjustment to the tool path to reduce the magnitude of errors. We call this directed generating. From the surface measurement, we create an error map in SAGUARO, subtract it from the ideal surface, and use the modified surface to generate a tool path. This process will converge on a surface whose accuracy is limited by the accuracy of measurements and the repeatability of the various sources of surface error.

\subsection{Automated calibration of tool motion}

The LOG's horizontal and vertical encoders measure the positions of the carriages that ride on bearing surfaces. The tool is displaced from the carriages by about $1 \mathrm{~m}$ horizontally and $2 \mathrm{~m}$ vertically, so the tool position is sensitive not only to carriage displacements but also to rotations at the $10 \mu \mathrm{rad}$ level. The new software has a feature we call Autocal, which uses a laser tracker to measure the tool position over a grid measuring $5 \mathrm{~m}$ horizontally ( $x$ direction) and $0.7 \mathrm{~m}$ vertically ( $z$ direction). We measure significant repeatable errors in $z$ vs $x, z$ vs $z$, and $x$ vs $x$. These errors include misalignment between the linear axes and the turntable axis that defines the $z$ axis. We use the laser tracker measurements to build a look-up table for errors in $x$ and $z$ as a function of the encoder readings, and apply a correction continuously in the control software.

\subsection{Safety features}

Safety to personnel and mirrors was a high priority in the LOG upgrade. A number of existing safety features were enhanced, including comparison of redundant encoders and monitoring of spindle motor current. An envelope is defined for each mirror and for each tool. The software uses these envelopes to prevent collisions with the mirror. A set of increasingly stringent tool speed limits are imposed as the distance between tool and mirror decreases. During a cut, if any monitored value exceeds its threshold, it triggers a controlled stop with the tool moving away from the mirror before motions are disabled.

\subsection{Finer diamonds}

With the improved accuracy of the upgraded LOG, it is worthwhile to finish the surface with finer diamonds in order to reduce subsurface damage proportionally. We then have less glass to remove in subsequent fine grinding (lapping) operations. We introduced a new generating tool with $30-40 \mu \mathrm{m}$ diamonds in a resin bond. Using a conservative criterion for subsurface damage, we need to remove only $100 \mu \mathrm{m}$ of glass after generating. We can do this in about 50 hours of fine grinding in the course of figuring the mirror from the $\sim 10 \mu \mathrm{m}$ rms generated surface to the $\sim 1 \mu \mathrm{m}$ rms initially polished surface.

\subsection{Operator platforms}

We replaced the operator platforms on both ends of the LOG bridge to provide more space, better access to the mirror, and better access to the tool where it contacts the mirror. 


\subsection{Testing the upgraded LOG}

Before putting a mirror on the LOG, we ran the LOG through over 90 tests of low-level functionality (e. g. speeds and accelerations), performance (primarily accuracy of motion), and safety. We then tested the system by generating a $1.2 \mathrm{~m}$ flat mirror and a $2 \mathrm{~m}$ granite disk with a curved surface. Although the mirror was nominally flat, we demonstrated the directed generating capability by machining a $150 \mu \mathrm{m}$ peak-to-valley trefoil shape and measuring it. We finished with the fine 30-40 $\mu \mathrm{m}$ diamond tool and confirmed that it gave an excellent finish and low subsurface damage. We then machined the granite disk to demonstrate the tool motion needed to generate a GMT off-axis segment, creating a cylindrical surface with a sag of $10 \mathrm{~mm}$ and verifying the shape accuracy with a laser tracker.

\subsection{Generating the rear surface of Segment 3}

Following the first two phases of the LOG upgrade we generated the flat rear surface of GMT Segment 3 along with the edge, bevels and bosses. Generating the flat surface involves all machine axes, including vertical, in order to incorporate the calibration for $z$ vs $x$. The new system met all requirements. It was much more reliable and consistent as measured by the number of unplanned interruptions, no more than 2 per week over the last 8 weeks of generating the rear surface. After an initial setting of the tool position relative to the mirror, it repeated to $10 \mu \mathrm{m}$ for many repetitions of the same path until we changed to a different tool. The fine diamond wheel produced a smooth, accurate and partially transparent surface, consistent with exceptionally little subsurface damage.

\section{UPGRADE OF LARGE POLISHING MACHINE}

With the LOG ready to generate the front surface of GMT Segment 2, we are in the process of upgrading the Large Polishing Machine (LPM). The LPM was installed in 2003 and was used to polish the second LBT primary mirror, GMT Segment 1, and the LSST combined primary and tertiary mirrors. The upgrade will give us new polishing capabilities as well as modern, supportable computers and software.

\subsection{Improved orbital polishing}

The most significant improvement in polishing methods is an enhanced orbital polishing system. When we polished Segment 1 we found that our $1.2 \mathrm{~m}$ stressed lap polishing system was not as effective for the off-axis mirror as it had been for symmetric mirrors. ${ }^{[5]}$ The removal rate varied across the surface, probably because of inconsistent fit between the lap and the aspheric surface. We implemented an orbital polisher with passive tools up to $35 \mathrm{~cm}$ diameter and gradually built up its capabilities during the polishing of Segment 1 . The lap executes an orbital motion which gives a well-defined removal footprint, or tool influence function, independent of position on the mirror (except when the tool reaches the edge). The tool follows a spiral path over the surface. We can vary the dwell time as a function of position with a dynamic range of about 100, giving a similar dynamic range in removal. We developed software to optimize dwell vs position based on a map of surface errors. ${ }^{[18]}$

We are building a new orbital polisher to replace the first-generation system that we installed while polishing Segment 1. It will drive passive laps ranging in size from $60 \mathrm{~cm}$ diameter - for correction of large-scale figure errors - to $5 \mathrm{~cm}$ in the radial dimension for correction of small-scale radial structure near the edge. Unlike the original orbital polisher, the new one will control lap rotation as well as orbit. Controlling lap rotation angle allows us to minimize the required shape change as the lap moves across the aspheric surface, and avoid having the same part of the lap go off the edge of the mirror in every orbit.

\subsection{Orbital stressed lap}

We also added an orbital capability to the $1.2 \mathrm{~m}$ stressed lap and tested it on the rear surface of Segment 3 as shown in Figure 7. In our traditional stressed lap polishing system, the lap moves continuously over the mirror surface, touching all points on the surface several times per hour. The predicted removal depends on dwell, pressure and relative speed between lap and mirror. We vary the dwell as a function of radius on the mirror but not azimuth. The primary means of non-axisymmetric figuring is varying the polishing pressure as a function of position. Because of the lap rotation and rapid motion across the mirror, the lap must change shape at a high rate. The magnitude and rate of shape change are no worse for a GMT segment than for an LBT primary mirror, but the changes are not axisymmetric on the mirror. The lack of symmetry probably contributed to the difficulty we had getting consistent removal during the polishing of GMT Segment 1. 


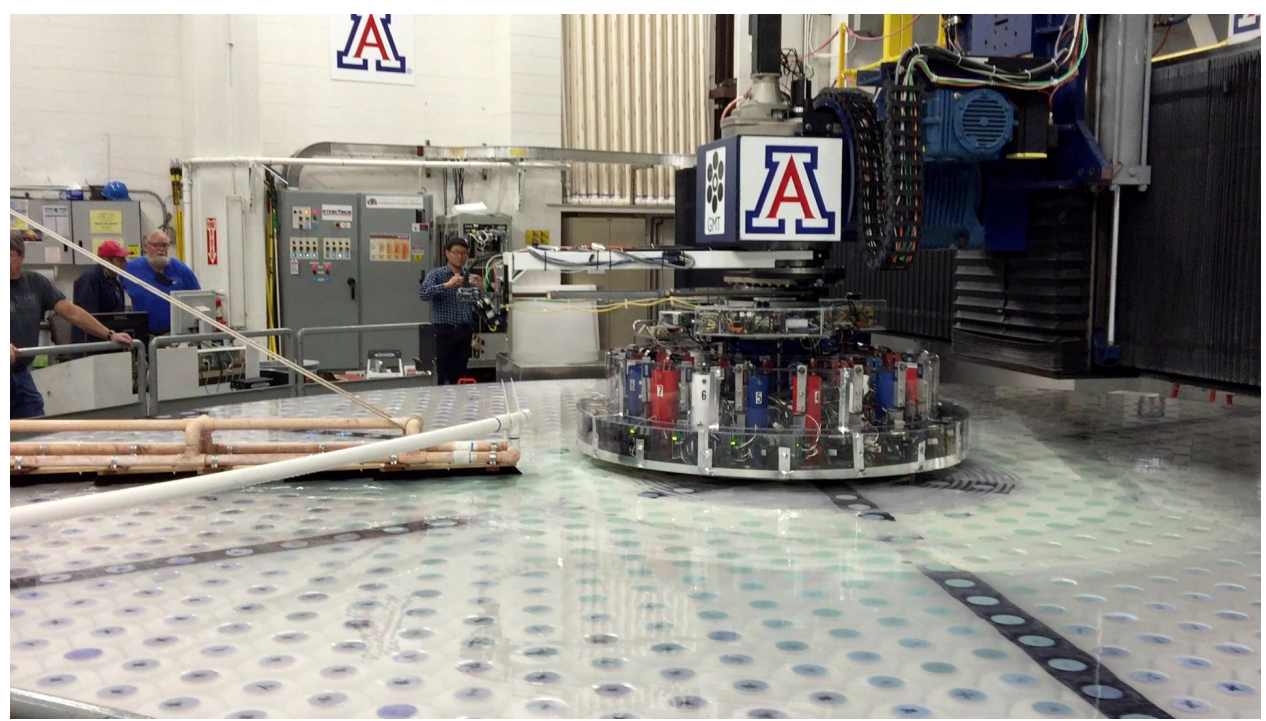

Figure 7. Orbital stressed lap during fine grinding of the rear surface of Segment 3. The working surface of the lap consists of Trizact diamond pads over a $1 \mathrm{~m}$ diameter. For this operation the lap orbited at $6 \mathrm{rpm}$ with a radius of $24 \mathrm{~cm}$. The 18 bending actuators, which control the shape of the lap for a curved surface, were disabled. The 3 lifting actuators were used to set the grinding pressure to $3400 \mathrm{~Pa}(0.5 \mathrm{psi})$.

We expect the orbital stressed lap to be more effective than the traditional stressed lap polishing motion for two reasons. First, orbital motion with dwell control provides at least 10 times greater dynamic range in removal than the traditional motion, more than enough to overcome any repeatable spatial variations in removal rate. Second, the motion of an orbital stressed lap - a rapid orbit with a radius up to $24 \mathrm{~cm}$ radius but otherwise very slow motion across the mirror surfacedoes not require rapid shape changes like our traditional stressed lap polishing with continuous lap rotation and largescale motion across the mirror. The slow shape changes provide much more time for pitch flow to eliminate shape errors in the lap as it moves over the aspheric surface. We therefore expect less spatial variation in removal rate.

\subsection{Optimization of dwell time vs position}

We are expanding the capabilities of the optimization software. The original program optimized the dwell time as a function of position for a single tool, based on a map of surface error. In many cases multiple tools of different sizes are needed to correct figure errors on different scales. The upgrade will allow simultaneous optimization for multiple tools, thereby determining the best way to combine tools of different sizes in a single polishing cycle.

\subsection{Fine grinding with Trizact diamond pads}

Between generating and polishing, we require a lapping process to remove the subsurface damage left from the generating tool. For all mirrors through the LSST mirror, we used traditional loose abrasive grinding with glass or ceramic tiles on the lap. While this process served the purpose of eliminating the subsurface damage and improving the figure from $\sim 20 \mu \mathrm{m} \mathrm{rms} \mathrm{to} \sim 1 \mu \mathrm{m} \mathrm{rms}$, the hard tiles presented a risk to the glass and we had several instances of minor damage to surfaces. We recently changed to using Trizact grinding pads with diamonds embedded in a soft polymer matrix. The soft pad material presents much less risk than hard tiles with loose abrasives. The bound diamonds remove glass faster than loose aluminum oxide abrasives of the same size, and the finer diamond grades such as $10 \mu \mathrm{m}$ leave so little subsurface damage that an $8 \mathrm{~m}$ mirror can be polished to a specular surface in about 50 hours. After a series of tests on small mirrors, and with a $1.2 \mathrm{~m}$ mirror on the LPM, we recently demonstrated the process on the rear surface of GMT Segment 3, shown in Figure 7. The next use will be on the front surface of Segment 2.

\section{UPGRADE OF OPTICAL TESTS}

The four major tests were listed in Section 3.2. They are complex systems, especially for the off-axis segments, and we experienced some delays in the assembly, testing and initial use for Segment 1. We had the interferometric test, pentaprism test, and LT+ in place before we polished Segment 1, but we were refining them throughout the process. The biggest challenges involved alignment of the interferometric test, which depends on wavefront measurements and laser 
tracker measurements of the position of the GMT segment and components of the null corrector over distances up to $24 \mathrm{~m}$. We experienced delays of several weeks during the initial alignment. We steadily improved and automated the alignment procedures until it took about 15 minutes by the end of the project. We also experienced serious challenges in correcting for image distortion in the interferometric test, limited spatial resolution of the interferometer, and a subtle instrumental effect that caused spurious slope errors in the pentaprism test. We eventually resolved these issues during the manufacture of Segment 1. Most importantly, the independent measurements agreed within the expected uncertainties and verified that Segment 1 was made to the right prescription.

One of the most serious limitations in the manufacture of Segment 1 was inadequate information about the figure at the edge of the mirror. We did not have a good way to measure the edge during fine grinding and therefore entered the polishing phase with large slope errors that made it impossible to acquire data with the interferometer. We were able to implement a new slope test, SCOTS, that has high spatial resolution ( $1 \mathrm{~cm}$ on the GMT segment), high accuracy on small scales, and high tolerance for slope errors, exactly what was needed to fill in the missing information at the edge. We then developed a method of combining the small-scale information from SCOTS with the large-scale information from the interferometric test. This method served to guide the figuring of Segment 1 to a final accuracy of $18 \mathrm{~nm} \mathrm{rms}$ surface after a simulated correction of low-order shape errors with active optics.

The interferometric test, especially the alignment procedure, is much more mature as we prepare to polish Segment 2. We will also have a mature SCOTS system to give accurate measurements of small-scale structure and regions with high slopes throughout the polishing process. During the fine grinding and initial polishing, we will use either the visiblewavelength SCOTS system or the IR version known as SLOTS - along with laser tracker measurements of large-scale structure - to control the edge and avoid the large slope errors that delayed progress on Segment 1.

We have continued to improve the tests during the interval between polishing Segment 1 and Segment 2. We have automated more of the alignment procedure to minimize user interactions that can disturb the alignment. We plan to replace the pentaprism camera with a model that eliminates the spurious slope errors. We are adding remotely controlled adjustments for the source and detector in SCOTS, which will serve to identify and average out certain sources of error. After these improvements, and because of the long interval between measuring Segment 1 and Segment 2, we plan to validate all tests - interferometric test, SCOTS, pentaprism test and LT+- by re-measuring Segment 1.

\section{CONCLUSION}

After polishing the LSST mirrors, upgrading the LOG, and generating the TAO mirror, the Mirror Lab is back at work on the GMT segments. We generated and polished the rear surfaces of Segments 2 and 3 and bonded the loadspreaders to Segment 2. We are preparing to generate the front surface of Segment 2. We had a successful casting of Segment 4, the center segment, and we are preparing to cast Segment 5. We added major capabilities to the fabrication and testing methods during the manufacture of Segment 1 and continue to improve these methods.

\section{ACKNOWLEDGEMENTS}

This work has been supported by the GMTO Corporation, a non-profit organization operated on behalf of an international consortium of universities and institutions: Astronomy Australia Ltd, the Australian National University, the Carnegie Institution for Science, Harvard University, the Korea Astronomy and Space Science Institute, the Smithsonian Institution, The University of Texas at Austin, Texas A\&M University, University of Arizona, University of Chicago, and the Sao Paulo Research Foundation (FAPESP).

This material is based in part upon work supported by AURA through the National Science Foundation under Scientific Program Order No. 10 as issued for support of the Giant Segmented Mirror Telescope for the United States Astronomical Community, in accordance with Proposal No. AST-0443999 submitted by AURA.

\section{REFERENCES}

[1] R. A. Bernstein, P. J. McCarthy, K. Raybould, B. C. Bigelow, A. H. Bouchez, J. M. Filgueira, G. H. Jacoby, M. Johns, D. Sawyer, S. Shectman and M. Sheehan, "Overview and status of the Giant Magellan Telescope project", in Ground-based and Airborne Telescopes V, ed. L. M. Stepp, R. Gilmozzi and H. J. Hall, Proc. SPIE 9145, 91451C (2014). 
[2] M. Johns, C. Hull, G. Muller, B. Irarrazaval, A. Bouchez, T. Chylek, C. Smith, A. Wadhavkar, B. Bigelow, S. Gunnels, B. McLeod and C. Buleri, "Design of the Giant Magellan Telescope", in Ground-based and Airborne Telescopes V, ed. L. M. Stepp, R. Gilmozzi and H. J. Hall, Proc. SPIE 9145, 91451F (2014).

[3] J. H. Burge, W. Davison, H. M. Martin and C. Zhao, "Development of surface metrology for the Giant Magellan Telescope primary mirror", in Advanced Optical and Mechanical Technologies in Telescopes and Instrumentation, ed. E. Atad-Ettedgui and D. Lemke, Proc. SPIE 7018, 701814 (2008).

[4] S. C. West, J. H. Burge, B. Cuerden, W. B. Davison, J. Hagen, H. M. Martin, M. T. Tuell and C. Zhao, "Alignment and use of the optical test for the $8.4 \mathrm{~m}$ off-axis primary mirrors of the Giant Magellan Telescope", in Modern Technologies in Space- and Ground-based Telescopes and Instrumentation, Proc. SPIE 7739, 77390N (2010).

[5] H. M. Martin, R. G. Allen, J. H. Burge, J. M. Davis, W. B. Davison, M. Johns, D. W. Kim, J. S. Kingsley, K. Law, R. D. Lutz, P. A. Strittmatter, P. Su, M. T. Tuell, S. C. West and P. Zhou, "Production of primary mirror segments for the Giant Magellan Telescope", in Advances in Optical and Mechanical Technologies for Telescopes and Instrumentation, ed. R. Navarro, C. Cunningham and A. Barto, Proc. SPIE 9151, 91510J (2014).

[6] H. M. Martin, R. G. Allen, J. H. Burge, D. W. Kim, J. S. Kingsley, K. Law, R. D. Lutz, P. A. Strittmatter, P. Su, M. T. Tuell, S. C. West and P. Zhou, "Production of $8.4 \mathrm{~m}$ segments for the Giant Magellan Telescope", in Modern Technologies in Space- and Ground-based Telescopes and Instrumentation II, ed. R. Navarro, C. R. Cunningham and E. Prieto, Proc. SPIE 8450, 84502D (2012).

[7] H. M. Martin, R. G. Allen, J. H. Burge, B. Cuerden, W. J. Gressler, W. Hubler, D. W. Kim, J. S. Kingsley, K. Law, P. A. Strittmatter, M. T. Tuell, S. C. West, C. Zhao and P. Zhou, "Manufacture of the combined primary and tertiary mirrors of the Large Synoptic Survey Telescope", in Advances in Optical and Mechanical Technologies for Telescopes and Instrumentation, ed. R. Navarro, C. Cunningham and A. Barto, Proc. SPIE 9151, 915125 (2014).

[8] P. Su, R. E. Parks, L. Wang, R. Angel and J. H. Burge, "Software configurable optical test system: a computerized reverse Hartmann test," Applied Optics, Vol 49, 4404-4412 (2010).

[9] P. Su, S. Wang, M. Khreishi, Y. Wang, T. Su, R. E. Parks, P. Zhou, M. Rascon, T. Zobrist, H. Martin and J. H. Burge, "SCOTS: A reverse Hartmann test with high dynamic range for Giant Magellan Telescope primary mirror segments", in Modern Technologies in Space- and Ground-based Telescopes and Instrumentation II, ed. R. Navarro, C. R. Cunningham and E. Prieto, Proc. SPIE 8450, 84500W (2012).

[10] P. Su, J. H. Burge, B. Cuerden and H. M. Martin, "Scanning pentaprism measurements of off-axis aspherics", in Advanced Optical and Mechanical Technologies in Telescopes and Instrumentation, ed. E. Atad-Ettedgui and D. Lemke, Proc. SPIE 7018, 70183T (2008).

[11] P. Su, J. H. Burge, B. Cuerden, R. G. Allen and H. M. Martin, "Scanning pentaprism measurements of off-axis aspherics II," in Optical Manufacturing and Testing VIII, ed. J. H. Burge, O. W. Fahnle and R. Williamson, Proc. SPIE 7426, 74260Y (2009).

${ }^{[12]}$ R. G. Allen, J. H. Burge, P. Su and H. M. Martin, "Scanning pentaprism test for the GMT 8.4 m off-axis segments", in Modern Technologies in Space- and Ground-based Telescopes and Instrumentation, Proc. SPIE 7739, 773911 (2010).

[13] T. Zobrist, J. H. Burge, W. Davison and H. M. Martin, "Measurement of large optical surfaces with a laser tracker", in Advanced Optical and Mechanical Technologies in Telescopes and Instrumentation, ed. E. Atad-Ettedgui and D. Lemke, Proc. SPIE 7018, 70183 U (2008).

[14] T. L. Zobrist, J. H. Burge and H. M. Martin, "Accuracy of laser tracker measurements of the GMT 8.4 m off-axis mirror segments", in Modern Technologies in Space- and Ground-based Telescopes and Instrumentation, Proc. SPIE 7739, 77390S (2010).

[15] D. Ketelsen, W. Davison, S. DeRigne and W. Kittrell, "A machine for complete fabrication of 8-m class mirrors", in Advanced Technology Optical Telescopes V, ed. L. M. Stepp, Proc. SPIE 2199, 651 (1994).

[16] J. M. Davis, H. M. Martin, D. W. Kim, A. R. Loeff, K. L. Kenagy, R. W. Sisk and J. R. Hagen, "Advances in diamond generating for 8.4 meter telescope mirrors", in Optifab 2015, ed. J. L. Bentley and S. Stoebenau, Proc. SPIE 9633, 96330Y (2015).

[17] G. A. Smith, B. J. Lewis, M. Palmer, D. W. Kim, A. R. Loeff and J. H. Burge, "Open source data analysis and visualization software for optical engineering", in Novel Optical Systems Design and Optimization XV, ed. G. G. Gregory and A. J. Davis, Proc. SPIE 8487, 84870F (2012).

[18] D. W. Kim, H. M. Martin and J. H. Burge, "Calibration and optimization of computer-controlled optical surfacing for large optics", in Optical Manufacturing and Testing IX, ed. J. H. Burge, O. W. Fähnle and R. Williamson, Proc. SPIE 8126, 812615 (2011). 